\title{
The Geographic Mosaic of Sex and Infection in Lake Populations of a New Zealand Snail at Multiple Spatial Scales
}

\author{
Daniela Vergara, ${ }^{1, \star}$ Curtis M. Lively, ${ }^{1}$ Kayla C. King, ${ }^{1, \dagger}$ and Jukka Jokela ${ }^{2,3}$ \\ 1. Department of Biology, Indiana University, Bloomington, Indiana 47405; 2. EAWAG, Swiss Federal Institute of Aquatic Science and \\ Technology, Überlandstrasse 133, 8600 Duebendorf, Switzerland; 3. ETH Zurich, Swiss Federal Institute of Technology, Institute of \\ Integrative Biology, 8092 Zurich, Switzerland
}

Submitted October 14, 2012; Accepted April 26, 2013; Electronically published August 20, 2013

Online enhancement: appendix. Dryad data: http://dx.doi.org/10.5061/dryad.7341c.

\begin{abstract}
AвSTRACт: Understanding how sexual and asexual forms of the same species coexist is a challenge for evolutionary biology. The Red Queen hypothesis predicts that sex is favored by parasite-mediated selection against common asexual genotypes, leading to the coexistence of sexual and asexual hosts. In a geographic mosaic, where the risk of infection varies in space, the theory also predicts that sexual reproduction would be positively correlated with disease prevalence. We tested this hypothesis in lake populations of a New Zealand freshwater snail, Potamopyrgus antipodarum, by comparing pairwise difference matrices for infection frequency and male frequency using partial Mantel tests. We conducted the test at three spatial scales: among lakes on the South Island, among depths within an intensively sampled lake (Lake Alexandrina), and within depths at Lake Alexandrina. We found that the difference in infection risk and the difference in the proportion of sexual snails were significantly and positively correlated at all spatial scales. Our results thus suggest that parasite-mediated selection contributes to the long-term coexistence of sexual and asexual individuals in coevolutionary hotspots, and that the "warmth" of hotspots can vary on small spatial scales.
\end{abstract}

Keywords: geographic mosaic theory of coevolution, host-parasite coevolution, Red Queen hypothesis, Mantel test, sexual reproduction.

One of the main aims of coevolutionary studies is to understand the strength of reciprocal selection over space and time (Thompson 2005). According to the geographic mosaic theory of coevolution, spatial and temporal variation in the strength of selection results in a geographic mosaic containing "hotspots" where interacting species are under reciprocal selection, and "coldspots" where selection is weak, absent, or not fully reciprocal (Thompson and Cunningham

\footnotetext{
* Corresponding author; e-mail: vergarad@indiana.edu.

${ }^{\dagger}$ Present address: Institute of Integrative Biology, University of Liverpool, Crown Street, Liverpool L69 7ZB, United Kingdom.
}

Am. Nat. 2013. Vol. 182, pp. 484-493. (C) 2013 by The University of Chicago. 0003-0147/2013/18204-54179\$15.00. All rights reserved.

DOI: $10.1086 / 671996$
2002; Forde et al. 2004; Thompson 2005; Laine 2006; Smith and Benkman 2007; Hanifin and Brodie 2008; Nash et al. 2008). The Red Queen hypothesis for the maintenance of sexual reproduction fits nicely into this framework provided there is geographic variation for the presence and/or strength of parasite-mediated selection against common host genotypes. Here, the prediction is that genetically diverse populations would be more likely to occur in geographic locations subject to predictable exposure to coevolving parasites, whereas parasite-free populations (or populations where the coevolutionary loop is blocked) would be expected to have reduced genetic diversity. If the selection imposed by coevolving parasites is sufficiently strong, then sexual reproduction can be favored over asexual reproduction (Jaenike 1978; Bell 1982; Hamilton 1982), even when there is a twofold cost of sex (Maynard Smith 1971, 1978; Williams 1975; Lively and Lloyd 1990). Hence, for species where asexual reproduction is possible, the expectation under the Red Queen hypothesis is that sexual reproduction would be more common in host populations engaged in coevolutionary interactions with virulent parasites, and asexual hosts would be more likely to occur in populations in which such interactions are not occurring (Hamilton 1980; Lloyd 1980). Empirical studies to date generally support this prediction (Glesener and Tilman 1978; Bell 1982; Burt and Bell 1987; Lively 1987; Lively and Jokela 2002), as well as other key assumptions of the Red Queen hypothesis (Busch et al. 2004; Decaestecker et al. 2007; Wolinska and Spaak 2009; Duneau et al. 2011; Luijckx et al. 2011). If instead sex is beneficial for reasons that are independent of coevolutionary interactions, no such association is expected.

The freshwater snail Potamopyrgus antipodarum is a useful organism to test these ideas, because geographically isolated populations vary in the frequencies of sexual and 


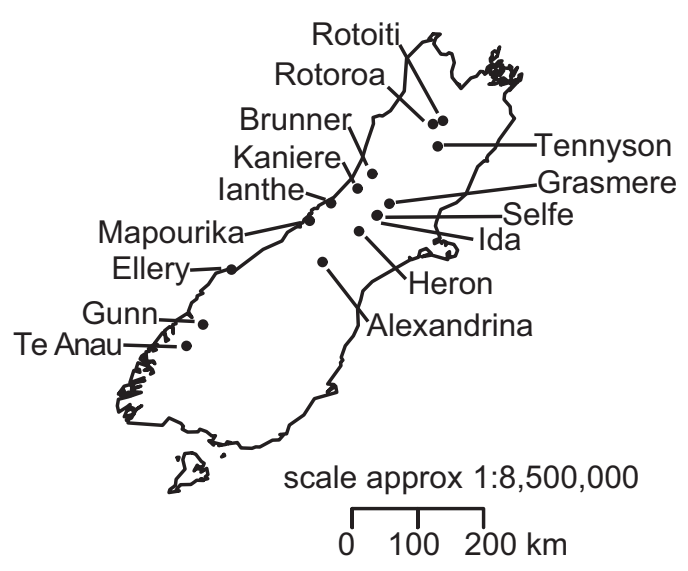

Figure 1: Map of the South Island of New Zealand showing the location of the 15 lakes sampled for this study.

asexual individuals. These snail populations also vary in the frequency of individuals that are sterilized by digenetic trematode parasites. Earlier work has shown that isolated populations having higher levels of infection also tend to have higher frequency of males (Lively 1987; Lively and Jokela 2002); this result holds whether or not males are included in the estimates of prevalence, suggesting that the pattern is not driven by overinfection of males (Lively 2001). Additional studies have shown further that a common trematode species, Microphallus, rapidly evolves to infect the most common local clonal lineages in two different field populations (Dybdahl and Lively 1998; Jokela et al. 2009), as well as in a controlled laboratory experiment (Koskella and Lively 2009). Here we present a more extensive and general test for a geographic mosaic in selection for sex by examining the frequency of infection and the frequency of sexually reproducing snails among samples collected across multiple spatial scales, which include much of the ecological variability present in the range of the interacting species. The results are consistent with a parasite-mediated, geographic mosaic of sex.

\section{Material and Methods}

\section{Study System}

Potamopyrgus antipodarum is a freshwater snail, which is native to New Zealand. Populations of this snail include either diploid, dioecious, sexual individuals or triploid, asexual females, or variable mixtures of both (Phillips and Lambert 1989; Wallace 1992; Dybdahl and Lively 1995). In addition, genetic data indicate that the triploid asexual females are derived from the local sexual populations, where sexual and asexual females coexist (Dybdahl and Lively 1995; Neiman et al. 2005). Potamopyrgus antipodarum also serves as a first intermediate host for at least 14 different parasite species, of which the sterilizing trematode Microphallus sp. is the most common in lake populations (Winterbourn 1974; Lively 1987; Hechinger 2012). In the well-studied population of Lake Alexandrina, both the prevalence of Microphallus infection and the proportion of sexual individuals declines with depth (Jokela and Lively 1995b), and these depth-specific clines have persisted for at least 2 decades (Jokela et al. 2009). A likely explanation for the association between high sex and infection in shallow habitats is that the final hosts for the parasite, waterfowl, often forage in shallow and mid-depth waters, but not in deeper areas

Table 1: Among-lakes generalized linear mixed model of variation in male frequency with respect to sampling depth (fixed factor), sampling year, and sampling site in the seven lakes included in the analysis

\begin{tabular}{|c|c|c|c|c|}
\hline & $F$ & df1 & $\mathrm{df} 2$ & $P$ \\
\hline \multicolumn{5}{|l|}{ Fixed effect: } \\
\hline \multirow[t]{2}{*}{ Depth } & 69.90 & 2 & 11,202 & $<.001$ \\
\hline & Estimate & $t$ value & df & Adjusted $P$ \\
\hline \multicolumn{5}{|l|}{ Pairwise contrast: } \\
\hline Shallow-midwater & .082 & 7.19 & 253 & $<.001$ \\
\hline Shallow-deep & .092 & 8.21 & 144 & $<.001$ \\
\hline \multirow[t]{2}{*}{ Midwater-deep } & .010 & 1.37 & 11,202 & .170 \\
\hline & Estimate & SE & $P$ & Low, high 95\% CI \\
\hline \multicolumn{5}{|l|}{ Random effects: } \\
\hline Year $\times$ site $($ lake $)$ & .966 & .202 & $<.001$ & $.641,1.455$ \\
\hline
\end{tabular}


Table 2: Among-lakes generalized linear mixed model of variation in prevalence of Microphallus with respect to sampling depth (fixed factor), sampling year, and sampling site in the seven lakes included in the analysis

\begin{tabular}{|c|c|c|c|c|}
\hline & $F$ & df1 & df2 & $P$ \\
\hline \multicolumn{5}{|l|}{ Fixed effects: } \\
\hline \multirow[t]{2}{*}{ Depth } & 12.31 & 2 & 8,408 & $<.001$ \\
\hline & Estimate & $t$ value & df & Adjusted $P$ \\
\hline \multicolumn{5}{|l|}{ Pairwise contrast: } \\
\hline Shallow-midwater & .004 & .56 & 6,022 & .577 \\
\hline Shallow-deep & .026 & 4.67 & 342 & $<.001$ \\
\hline \multirow[t]{2}{*}{ Midwater-deep } & .022 & 3.14 & 1,323 & .003 \\
\hline & Estimate & SE & $P$ & Low, high 95\% CI \\
\hline \multicolumn{5}{|l|}{ Random effects: } \\
\hline Year $\times$ site (lake) & 1.027 & .223 & $<.001$ & $.671,1.572$ \\
\hline
\end{tabular}

Note: Variation among years and sampling sites in different lakes were fit as a single random effect. The model was fit using a binomial response and logit link function (AICC 69985, BIC 69992). Significance of pairwise contrast was adjusted for multiple tests using a sequential Bonferroni correction. $\mathrm{CI}=$ confidence interval.

(Jokela and Lively 1995b). Thus, parasites infecting snails in the shallow-water margins of the lake are more likely to be consumed by waterfowl, which is required for completion of the parasite's life cycle. Parasite eggs deposited in deeper parts of the lake can be ingested by snails, but infected snails in deep areas are much less likely to be eaten by birds, thus breaking the coevolutionary loop. Direct experimental evidence for the idea that the shallow-water margins of lakes are coevolutionary hotspots is presented in King et al. (2009; 2011).

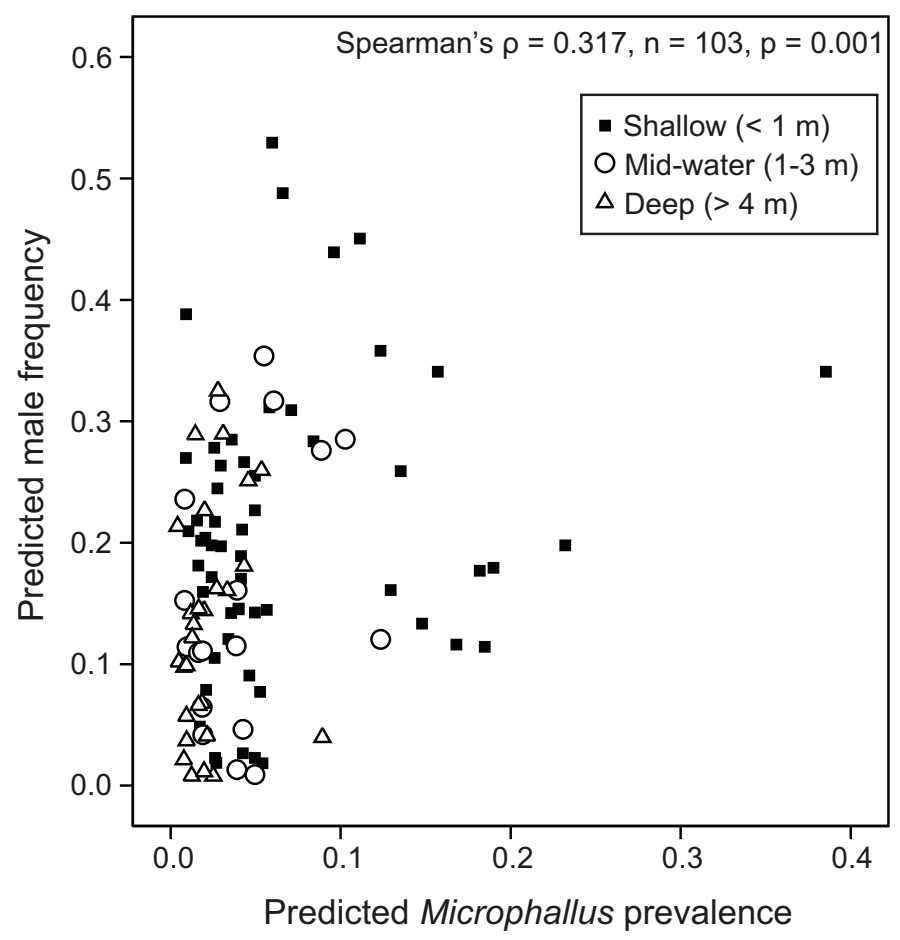

Figure 2: Scatterplot for the predicted values of male frequency against the predicted values for Microphallus prevalence for seven lakes on the South Island of New Zealand. The predicted values were calculated using generalized linear mixed models with binomial errors and logit-link function (see "Material and Methods"). 
Table 3: Within-Lake Alexandrina generalized linear mixed model of variation in male frequency with respect to sampling depth (fixed factor, three depths) and sampling site (20 shallow sites, 17 of those sampled also in midwater, and 15 in deep)

\begin{tabular}{|c|c|c|c|c|}
\hline & $F$ & df1 & df2 & $P$ \\
\hline \multicolumn{5}{|l|}{ Fixed effect: } \\
\hline \multirow[t]{2}{*}{ Depth } & 22.96 & 2 & 49 & $<.001$ \\
\hline & Estimate & $t$ value & df & Adjusted $P$ \\
\hline \multicolumn{5}{|l|}{ Pairwise contrast: } \\
\hline Shallow-midwater & .083 & 4.51 & 39 & $<.001$ \\
\hline Shallow-deep & .112 & 6.44 & 41 & $<.001$ \\
\hline \multirow[t]{2}{*}{ Midwater-deep } & .029 & 2.13 & 56 & .038 \\
\hline & Estimate & SE & $P$ & Low, high $95 \%$ CI \\
\hline \multicolumn{5}{|l|}{ Random effects: } \\
\hline Depth $\times$ site & .139 & .050 & .005 & $.069, .281$ \\
\hline
\end{tabular}

Note: Variation among samples was fit by including the depth $\times$ site interaction as a single random effect. The model was fit using a binomial response and logit link function (AICC 27591, BIC 27598). Significance of pairwise contrast was adjusted for multiple tests using a sequential Bonferroni correction. $\mathrm{CI}=$ confidence interval.

\section{Data Collection}

We analyzed samples collected between 1985 and 2011 from 15 lakes on the South Island of New Zealand (fig. 1, table A1; tables A1-A3 available online). The data set includes 219 random samples totaling 22,794 dissected snail individuals. For each snail, we determined the gender, size, and parasite infection status. In 10 lakes, we sampled three depths, shallow $(<1 \mathrm{~m})$, midwater $(1-3 \mathrm{~m})$ and deep $(>4 \mathrm{~m})$; for the other five lakes, we sampled only the shallow and deep regions (table A1). Some of the data came from previous studies, but the majority of the data are reported here for the first time (table A1). Our data are available from the Dryad Digital Repository, http:// dx.doi.org/10.5061/dryad.7341c; Vergara et al. 2013.

Table 4: Within-lake Alexandrina generalized linear mixed model of variation in prevalence of Microphallus infection with respect to sampling depth (fixed factor, three depths) and sampling site (20 shallow sites, 17 of those sampled also in midwater, and 15 in deep)

\begin{tabular}{lcccc}
\hline & $F$ & df1 & df2 & $P$ \\
\hline Fixed effect: & & & & \\
Corrected model & 47.45 & 3 & 82 & $<.001$ \\
Depth & 22.96 & 2 & 49 & $<.001$ \\
Snail length & 97.23 & 1 & 5,347 & $<.001$ \\
\cline { 2 - 5 } & Estimate & $t$ value & df & Adjusted $P$ \\
\cline { 2 - 5 } & & & & \\
Pairwise contrast: & .157 & 3.85 & 37 & .001 \\
Shallow-midwater & .176 & 4.40 & 37 & $<.001$ \\
Shallow-deep & .019 & 2.29 & 53 & .026 \\
Midwater-deep & Estimate & SE & $P$ & Low, high 95\% CI \\
\cline { 2 - 5 } & & & & $.796,2.154$ \\
Random effects: & 1.310 & .332 & $<.001$ & \\
Depth $\times$ site &
\end{tabular}

Note: Length of individual snails was used as a covariate; the significant result shows that the prevalence of infection increases with snail size. Variation among samples was fit by including depth $\times$ site interaction as a single random effect. The model was fit using a binomial response and logit link function (AICC 33340, BIC 33347). Significance of pairwise contrast was adjusted for multiple tests using a sequential Bonferroni correction. $\mathrm{CI}=$ confidence interval. 


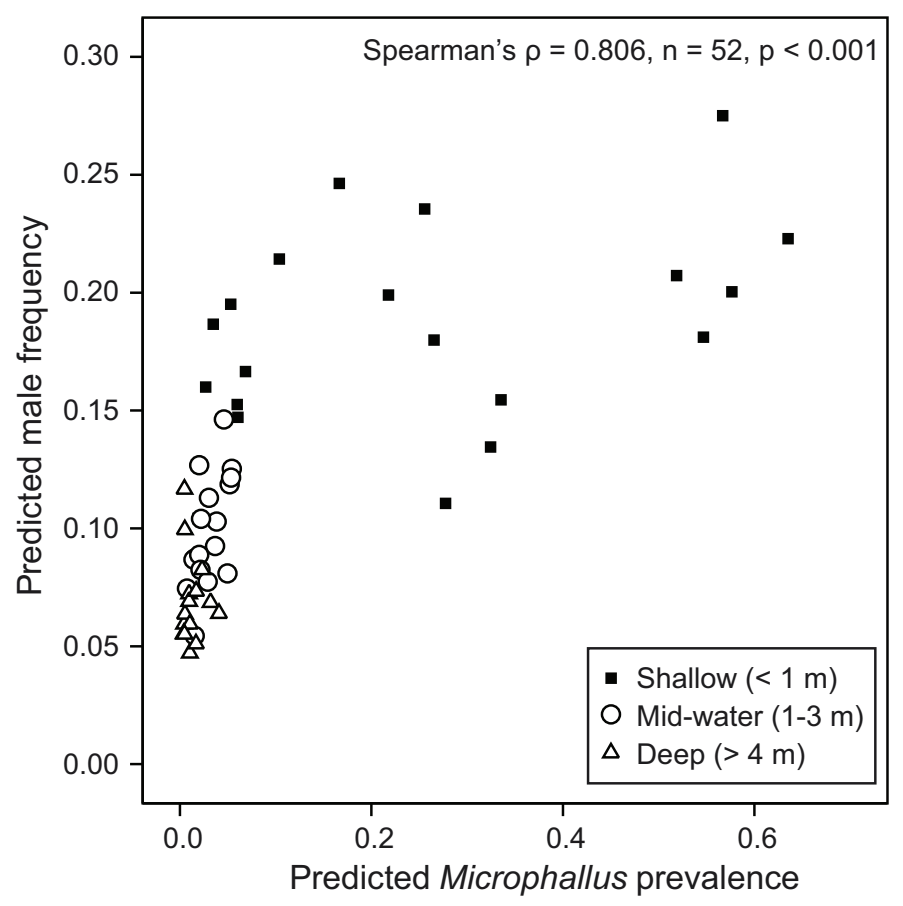

Figure 3: Scatterplot for the predicted values of male frequency against the predicted values for Microphallus prevalence among sites for three habitats (shallow, mid-depth, deep) within Lake Alexandrina. The predicted values were calculated using generalized linear mixed models with binomial errors and logit-link function (see "Material and Methods").

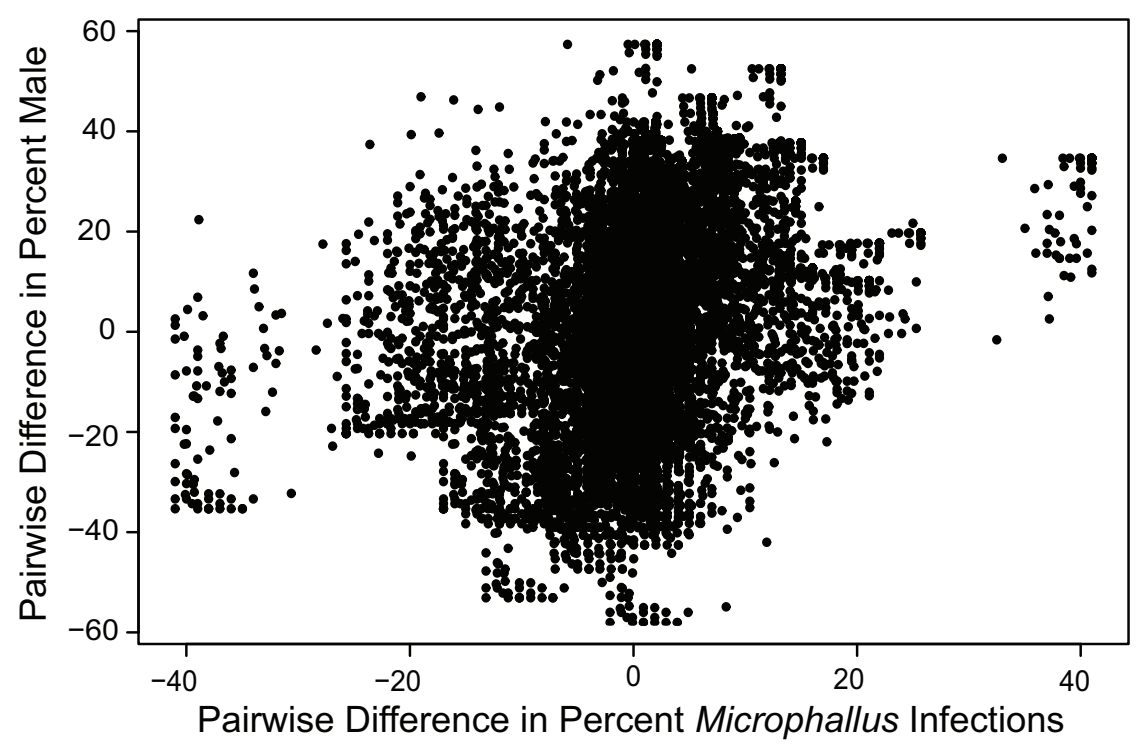

Figure 4: Pairwise comparisons among lakes. Pairwise difference in percent males ( $Y$-axis), against the pairwise difference in percent Microphallus infection ( $X$-axis), in the shallow, mid-depth, and deep habitats for every sample from 14 lakes. Each point represents a pairwise difference between each year, site, and depth from every lake ( $N=167$ samples). 


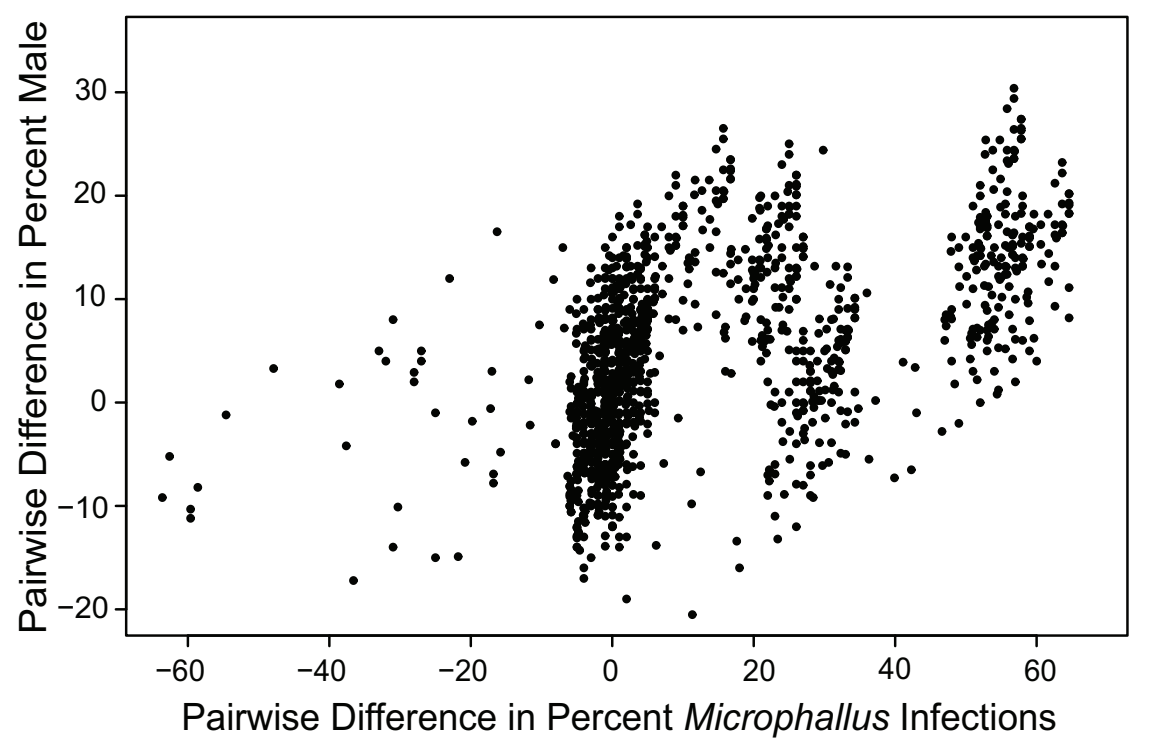

Figure 5: Pairwise comparisons within Lake Alexandrina. Pairwise differences in percent males ( $Y$-axis), against the pairwise difference in percent Microphallus infections ( $X$-axis), including all sites from the shallow (20 sites), mid-depth (17 sites), and deep (15 sites) habitats.

\section{Data Analysis}

In the first set of analyses, we examined variation in male frequency and the prevalence of infection using generalized linear mixed models (GLMMs), where our aim was to test the hypothesis that shallow-water habitats are coevolutionary hotspots. We analyzed both (1) among-lake variation and (2) within-lake variation among sites at Lake Alexandrina. For both spatial scales, we fit a GLMM predicting the probability of observing a male and another GLMM predicting probabilities of observing a Microphallus infection correcting for random effects (e.g. lake identity, sampling site, sampling year).

In the among-lakes GLMMs, we included lakes where sexual and asexual snails coexist and for which we had samples from three depths ( 7 out of the 15 lakes, table A1). In these models, we used sampling depth (shallow, midwater, deep) as a fixed factor, and the interaction between year and sampling site nested within lake as a random factor, which fits a random effect for each year $x$ site combination (65 random effects). After fitting the GLMMs, we then calculated the Spearman correlation coefficient between predicted male frequency and predicted Microphallus prevalence.

We analyzed samples from Lake Alexandrina separately. In the year 2000, we sampled 20 sites for shallow, 17 for midwater, and 15 for deep and were therefore able to conduct a detailed analysis of within-lake patterns in male frequency and the frequency of Microphallus infection. In this analysis, we included the sampling site $x$ depth interaction (sample location) as a random factor, and we used depth as a fixed factor. In addition, we used snail shell length as a covariate in the analysis of the frequency of Microphallus infection. As above, we used the Spearman rank correlation to test for an association between predicted male frequency and predicted prevalence of infection. GLMM analyses were conducted using GENLINMIXED procedure of IBM SPSS statistics, version 19.

In the second set of analyses, we used partial Mantel tests to examine the correlation between male frequency and the prevalence of Microphallus infection among lakes, while correcting for the geographic distance between the lakes. More specifically, we examined the correlation between the matrix of male frequencies and the matrix of infection prevalence, correcting for the matrix of geographic distances between samples, using the "vegan" package in R software (Oksanen et al. 2013). We included all samples from all years that were available from all lakes, except Lake Alexandrina, which we analyzed separately. For the within-lake analysis of Lake Alexandrina, we first examined the correlation between the matrix of male frequency and the matrix of infection prevalence, correcting for distance (using the partial mantel test) across all sites and depths. We then conducted the same test separately within each of the three depth habitats.

\section{Results}

Using the GLMM, we found that the shallow-water margins of lakes had a significantly higher frequency of males than the midwater and deep habitats (mean \pm SE: 


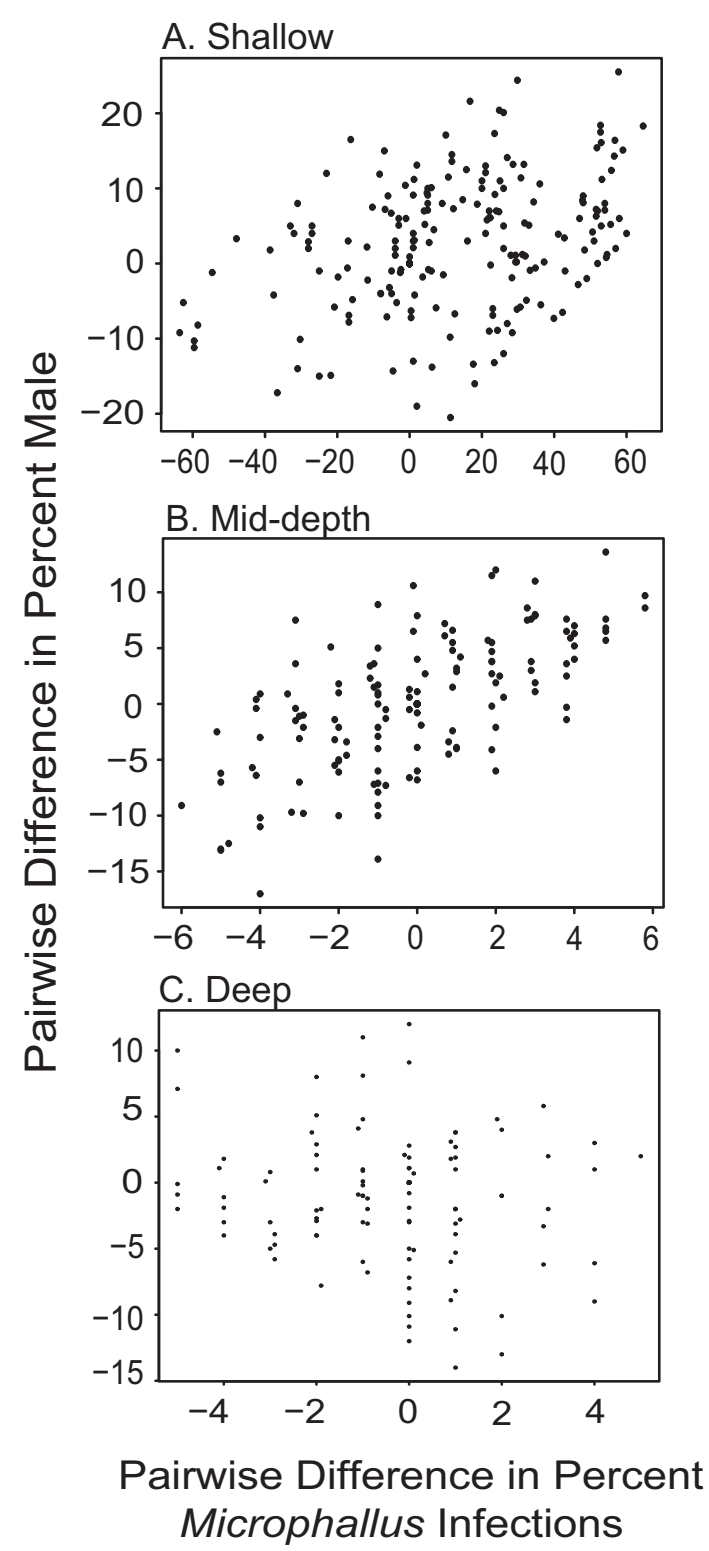

Figure 6: Pairwise comparisons within the three depth-related habitats of Lake Alexandrina. Each of the panels shows the pairwise difference in percent males ( $Y$-axis) against the pairwise difference in percent Microphallus infections ( $X$-axis) for the different depths (habitats) sampled. $A$, shallow (20 sites); $B$, mid-depth (17 sites); and $C$, deep (15 sites).

$17.5 \% \pm 1.9 \%$ vs. $9.4 \% \pm 1.2 \%$ and $8.3 \% \pm 1.1 \%$, respectively, for shallow, midwater, and deep samples; table 1). We also found that prevalence of Microphallus infections was higher in shallow and midwater regions than in the deep habitats of the lakes (mean \pm SE: $4.8 \% \pm$ $0.7 \%, 4.4 \% \pm 0.8 \%$ and $2.2 \% \pm 0.4 \%$, respectively, for shallow, midwater, and deep samples; table 2). The same result was obtained for the shallow-deep comparison when males were excluded from the analysis (table A2); thus, the overall pattern is not driven by differential male susceptibility. The correlation between the predicted values of male frequency and Microphallus prevalence was positive and statistically significant (Spearman's $\rho=0.317$, $N=103, P=.001$, fig. 2), and the result held whether or not males were included in the estimates of infection prevalence (Spearman's $\rho=0.340, N=103, P=.001$ ). These findings indicate that the shallow regions of lakes have a higher male frequency as well as a higher Microphallus prevalence. They also show that male frequency and Microphallus prevalence are positively correlated as predicted by the coevolutionary hotspot/Red Queen hypothesis.

Analysis among sites and habitats within Lake Alexandrina yielded similar results, suggesting that the shallowwater habitats are coevolutionary hotspots on both large and small geographic scales. Male frequency in the shallow habitat was almost twofold greater than that of the midwater habitat (mean \pm SE: $18.0 \% \pm 1.5 \%$ vs. $9.7 \% \pm$ $1.1 \%$, respectively, for shallow and midwater; table 3 ). The difference was even greater between the shallow and deep habitats $(18.0 \% \pm 1.5 \%$ in shallow vs. $6.7 \% \pm 0.9 \%$ in deep). For Microphallus prevalence, the difference between shallow and the two other depths was more than fivefold (length-adjusted mean \pm SE: $18.3 \% \pm 4.0 \%, 2.6 \% \pm$ $0.8 \%$, and $0.7 \% \pm 0.3 \%$, respectively, for shallow, midwater, and deep samples; table 4). Virtually the same result was obtained when males were excluded from the prevalence estimate (table A3). As for the above analysis among lakes, the correlation between predicted male frequency and predicted prevalence of Microphallus infection was positive and statistically significant (Spearman's $\rho=$ $0.806, n=52, P<.001$; fig. 3 ). The result also held when males were removed from the estimates of infection prevalence (Spearman's $\rho=0.808, N=52, P<.001$ ).

The partial Mantel test for all samples $(N=167)$ collected from 14 lakes showed that even when the variation among years, as well as sampling sites and sampling depth were not included in the analysis, male frequency and the prevalence of Microphallus infection were positively correlated $(r=0.3282, P<.001$, correcting for geographic distance; fig. 4), even when males were excluded from the estimates of infection prevalence $(r=0.3505, P<.001)$. When we pooled the samples within each of the different habitats for all 15 lakes (including Lake Alexandrina) from each year and site, we also observed a significant and positive correlation $(r=0.339, P<.005$, correcting for geographic distance; fig. A1, available online). The same result was obtained when males were not included in the estimates of infection prevalence $(r=0.347, P<.005)$.

The partial Mantel test for the within Lake Alexandrina 
samples $(N=52)$ showed an even stronger positive correlation between male frequency and Microphallus prevalence ( $r=0.543, P<.005$, correcting for geographic distance; fig. 5; when males were excluded from estimates of prevalence: $r=0.508, P<.005)$. When we separately analyzed the samples from each depth of Lake Alexandrina, we found that the correlation between male frequency and infection prevalence was positive and statistically significant in both the shallow and midwater habitats but not in the deep habitat $(r=0.362, P<.005 ; r=0.653, P<$ $.005 ; r=-0.15, P=.920$, respectively, for shallow, midwater, and deep habitats; fig. 6). The results were essentially identical when males are removed from the estimates of infection prevalence $(r=0.351, P<.005 ; r=0.572, P<$ $.005 ; r=-0.083, P=.838$, respectively). Thus, for all the analyses, the patterns that we observed within and among lakes cannot be explained by differential susceptibility of male and female hosts.

\section{Discussion}

We evaluated the confluence between the Red Queen hypothesis and the geographic mosaic theory of coevolution by examining the differences in prevalence of infection and the frequency of males at different spatial scales. These spatial scales covered a range of depths within and between lake populations of the New Zealand snail, Potamopyrgus antipodarum. Using partial Mantel tests, we observed whether, independent of the geographic distance, there was a positive correlation between the difference in infection frequency and the difference in male frequency as predicted by the parasite hypothesis for sex.

Taking together the analysis of 14 New Zealand lakes and specifically of Lake Alexandrina, we found that the pattern of parasitism and sexual reproduction holds over large and small geographic scales. The comparison within similar depths strengthens our argument that the difference in percent Microphallus infection is positively and significantly related to the difference in percent males, independent of depth differences per se (fig. 4). The independent analyses of the three different depths in Lake Alexandrina support the idea that the shallow and mid-depth are regions where coevolutionary interactions are occurring, while the deep habitat appears to be a coevolutionary coldspot (fig. 6). This finding supports studies showing that sexual reproduction should be found in regions where hosts are engaged in coevolutionary interactions with virulent parasites, while parthenogenetic reproduction should dominate regions where the parasite burden is low (Glesener and Tilman 1978; Hamilton 1980, 1982; Lloyd 1980; Lively 1992; Lively and Jokela 2002). These results also suggest that geographic distance, as such, does not play a major role in explaining the pattern of male presence. On the other hand, parasites do play a role in explaining the frequency of males independent of the geographic location.

According to the geographic mosaic theory of coevolution, variation in the strength of selection generates mosaics of coevolutionary hotspots and coldspots across landscapes (Thompson 1994, 1999, 2005). The geographic mosaic theory of coevolution is supported by empirical studies showing that selection intensity changes in magnitude between coldspots and hotspots (Forde et al. 2004; Smith and Benkman 2007; Hanifin and Brodie 2008; Nash et al. 2008; Smith et al. 2011) and that the mosaic can occur at small spatial scales (Laine 2006). The geographic mosaic theory of coevolution also predicts that coevolved traits can show clines across landscapes (Thompson 1999). Previous work in Lake Alexandrina showed a correlation between the frequency of males and the prevalence of $\mathrm{Mi}$ crophallus infections in the shallow and deep regions of the lake (Jokela and Lively 1995a; Jokela et al. 2009; King et al. 2009, 2011). Here we show that even among different sites in the same lake, and at the same depth, the predicted pattern exists between male frequency and prevalence of infection, suggesting that a coevolutionary mosaic can exist in the face of migration on very small spatial scales (fig. 6). In other words, there seems to be variation in the "warmth" of the hotspots.

In conclusion, the results show that the pairwise difference in the percentage of sexual individuals is positively and significantly correlated with the pairwise difference in percent infection by a highly virulent parasite. This positive correlation was found at various spatial scales: across lakes on the South Island of New Zealand, as well as among and within the different depths of Lake Alexandrina. These results, suggest that, regardless of the geographic scale, the difference in parasite prevalence explains the difference in sexual reproduction, which is consistent with the Red Queen hypothesis.

\section{Acknowledgments}

We thank J. Van Berkel for his assistance at the University of Canterbury field station in Kaikoura, New Zealand, S. Dickinson and T. Jackson for the statistical advice, P. J. Wu for the programming support, and F. Bashey-Visser, C. Lorenzi, and L. Morran for comments on the manuscript. The authors acknowledge financial support from National Science Foundation grants DEB-0640639 to C.M.L. and J. J. and DDIG-1110437 to D.V., a Royal Society Newton International fellowship to K.C.K., the Swiss National Science Foundation to J.J., and a National Geographic Society (883510) award to D.V. 


\section{Literature Cited}

Bell, G. 1982. The masterpiece of nature: the evolution and genetics of sexuality. University of California Press, Berkeley.

Burt, A., and G. Bell. 1987. Mammalian chiasma frequencies as a test of two theories of recombination. Nature 326:803-805.

Busch, J. W., M. Neiman, and J. M. Koslow. 2004. Evidence for the maintenance of sex by pathogens in plants. Evolution 58:25842590.

Decaestecker, E., S. Gaba, J. A. M. Raeymaekers, R. Stoks, L. Van Kerckhoven, D. Ebert, and L. De Meester. 2007. Host-parasite "Red Queen” dynamics archived in pond sediment. Nature 450:870874.

Duneau, D., P. Luijckx, F. Ben-Ami, C. Laforsch, and D. Ebert. 2011. Resolving the infection process reveals striking differences in the contribution of environment, genetics and phylogeny to host-parasite interactions. BMC Biology 9:11-22.

Dybdahl, M. F., and C. M. Lively. 1995. Diverse, endemic and polyphyletic clones in mixed populations of a freshwater snail (Potamopyrgus antipodarum). Journal of Evolutionary Biology 8:385398.

-1998. Host-parasite coevolution: evidence for rare advantage and time-lagged selection in a natural population. Evolution 52: 1057-1066.

Forde, S. E., J. N. Thompson, and B. J. M. Bohannan. 2004. Adaptation varies through space and time in a coevolving host-parasitoid interaction. Nature 431:841-844.

Glesener, R. R., and D. Tilman. 1978. Sexuality and the components of environmental uncertainty: clues from geographical parthenogenesis in terrestrial animals. American Naturalist 112:659-673.

Hamilton, W. D. 1980. Sex versus non-sex versus parasite. Oikos 35 : 282-290.

-1982. Pathogens as causes of genetic diversity in their host populations. Pages 269-296 in R. M. Anderson, and R. M. May, eds. Population biology of infectious diseases. Springer, New York.

Hanifin, C. T., E. D. Brodie Jr., and E. D. Brodie III. 2008. Phenotypic mismatches reveal escape from arms-race coevolution. PLoS Biology 6:471-482.

Hechinger, R. F. 2012. Faunal survey and identification key for the trematodes (Platyhelminthes: Digenea) infecting Potamopyrgus antipodarum (Gastropoda: Hydrobiidae) as first intermediate host. Zootaxa 3418:1-27.

Jaenike, J. 1978. A hypothesis to account for the maintenance of sex within populations. Evolutionary Theory 3:191-194.

Jokela, J., M. F. Dybdahl, and C. M. Lively. 1999. Habitat-specific variation in life-history traits, clonal population structure, and parasitism in a freshwater snail (Potamopyrgus antipodarum). nal of Evolutionary Biology 12:350-360.

. 2009. The maintenance of sex, clonal dynamics, and hostparasite coevolution in a mixed population of sexual and asexual snails. American Naturalist 174(suppl.):S43-S53.

Jokela, J., and C. M. Lively. 1995a. Parasites, sex, and early reproduction in a mixed population of freshwater snails. Evolution 49: 1268-1271.

-1995b. Spatial variation for infection by digenetic trematodes in a population of freshwater snails (Potamopyrgus antipodarum). Oecologia (Berlin) 103:509-517.

King, K. C., L. F. Delph, J. Jokela, and C. M. Lively. 2009. The geographic mosaic of sex and the Red Queen. Current Biology 19: $1438-1441$.
- 2011. Coevolutionary hotspots and coldspots for host sex and parasite local adaptation in a snail-trematode interaction. kos 20:1335-1340.

Koskella, B., and C. M. Lively. 2009. Evidence for negative frequencydependent selection during experimental coevolution of a freshwater snail and a sterilizing trematode. Evolution 63:2213-2221.

Laine, A.-L. 2006. Evolution of host resistance: looking for coevolutionary hotspots at small spatial scales. Proceedings of the Royal Society B: Biological Sciences 273:267-273.

Lively, C. M. 1987. Evidence from a New Zealand snail for the maintenance of sex by parasitism. Nature 328:519-521.

- 1992. Parthenogenesis in a freshwater snail: reproductive assurance versus parasitic release. Evolution 46:907-913.

. 2001. Trematode infection and the distribution and dynamics of parthenogenetic snail populations. Parasitology 123:S19-S26.

Lively, C. M., and J. Jokela. 2002. Temporal and spatial distributions of parasites and sex in a freshwater snail. Evolutionary Ecology Research 4:219-226.

Lively, C. M., and D. G. Lloyd. 1990. The cost of biparental sex under individual selection. American Naturalist 135:489-500.

Lloyd, D. G. 1980. Benefits and handicaps of sexual reproduction. Evolutionary Biology 13:69-111.

Luijckx, P., F. Ben-Ami, L. Mouton, L. Du Pasquier, and D. Ebert. 2011. Cloning of the unculturable parasite Pasteuria ramosa and its Daphnia host reveals extreme genotype-genotype interactions. Ecology Letters 14:125-131.

Maynard Smith, J. 1971. What use is sex? Journal of Theoretical Biology 30:319-335.

. 1978. The evolution of sex. Cambridge University Press, Cambridge.

Nash, D. R., T. D. Als, R. Maile, G. R. Jones, and J. J. Boomsma. 2008. A mosaic of chemical coevolution in a large blue butterfly. Science 319:88-90.

Neiman, M., J. Jokela, and C. M. Lively. 2005. Variation in asexual lineage age in Potamopyrgus antipodarum, a New Zealand snail. Evolution 59:1945-1952.

Oksanen, J., F. G. Blanchet, R. Kindt, P. Legendre, P. R. Minchin, R. O'Hara, G. L. Simpson, P. Solymos, M. H. H. Stevens, and H. Wagner. 2013. Community ecology package “vegan." http:// vegan.r-forge.r-project.org.

Phillips, N. R., and D. M. Lambert. 1989. Genetics of Potamopyrgus antipodarum (Gastropoda, Prosobranchia): evidence for reproductive modes. New Zealand Journal of Zoology 16:435-445.

Smith, D. L., L. Ericson, and J. J. Burdon. 2011. Co-evolutionary hot and cold spots of selective pressure move in space and time. Journal of Ecology 99:634-641.

Smith, J. W., and C. W. Benkman. 2007. A coevolutionary arms race causes ecological speciation in crossbills. American Naturalist 169: 455-465.

Thompson, J. N. 1994. The coevolutionary process. University of Chicago Press, Chicago.

- 1999. Specific hypotheses on the geographic mosaic of coevolution. American Naturalist 153(suppl.):S1-S14.

- 2005. The geographic mosaic of coevolution. University of Chicago Press, Chicago.

Thompson, J. N., and B. M. Cunningham. 2002. Geographic structure and dynamics of coevolutionary selection. Nature 417:735738.

Vergara, D., C. M. Lively, K. C. King, and J. Jokela. Data from: The geographic mosaic of sex and infection in lake populations 
of a New Zealand snail at multiple spatial scales. American Naturalist, Dryad Digital Repository, http://dx.doi.org/10.5061/ dryad.7341c.

Wallace, C. 1992. Parthenogenesis, sex, and chromosomes in Potamopyrgus. Journal of Molluscan Studies 58:93-107.

Williams, G. C. 1975. Sex and evolution. Princeton University Press, Princeton, NJ.

Winterbourn, M. J. 1974. Larval Trematoda parasitizing the New
Zealand species of Potamopyrgus (Gastropoda: Hydrobiidae). Mauri Ora 2:17-30.

Wolinska, J., and P. Spaak. 2009. The cost of being common: evidence from natural Daphnia populations. Evolution 63:1893-1901.

Associate Editor: Kimberly A. Hughes Editor: Mark A. McPeek

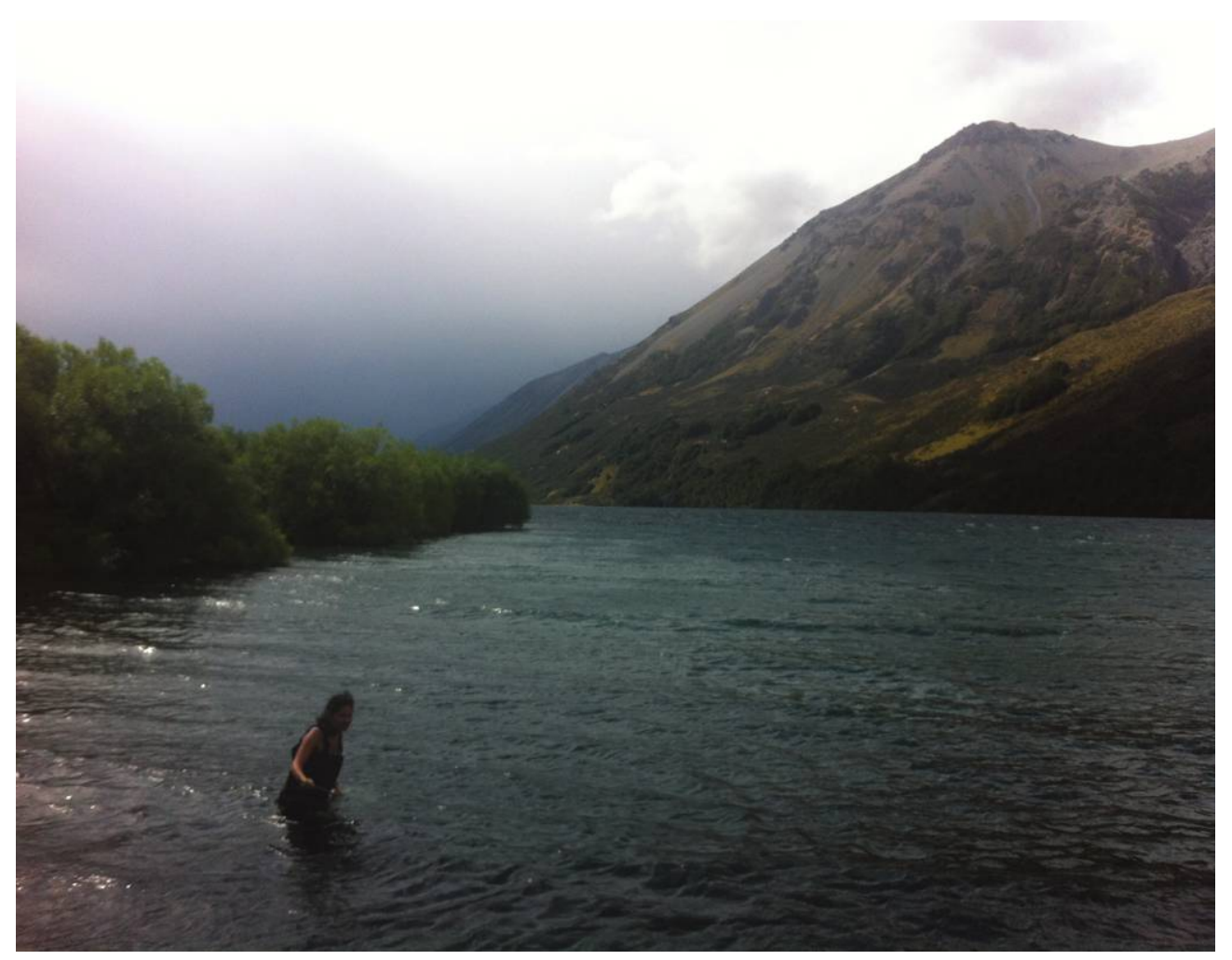

Daniela Vergara at Lake Selfe in the Canterbury Region of New Zealand's South Island. Photo credit: Andrew McKinney. 